\title{
Original Research Cariology \\ Fluoride retention in saliva and in dental biofilm after different home-use fluoride treatments
}

\author{
Daniela Correia Cavalcante \\ SOUZA \\ Marisa MALTZ \\ Lina Naomi HASHIZUME
}

Department of Preventive and Social Dentistry, Faculty of Dentistry, Universidade Federal do Rio Grande do Sul - UFRGS, Porto Alegre, RS, Brazil.
Declaration of Interests: The authors certify that they have no commercial or associative interest that represents a conflict of interest in connection with the manuscript.

\section{Corresponding Author:}

Lina Naomi Hashizume

E-mail: Ihashizume@yahoo.com

DOI: 10.1590/1807-3107BOR-2014.vol28.0039 Epub XXXXX, 2014

Submitted: May 17, 2013 Accepted for publication: Apr 22, 2014 Last revision: Aug 01, 2014

\begin{abstract}
This single-blind, randomized, crossover study aimed at assessing the long-term fluoride concentrations in saliva and in dental biofilm after different home-use fluoride treatments. The study volunteers $(n=38)$ were residents of an area with fluoridated drinking water. They were administered four treatments, each of which lasted for one week: twice-daily placebo dentifrice, twice-daily fluoride dentifrice, twice-daily fluoride dentifrice and once-daily fluoride mouthrinse, and thrice-daily fluoride dentifrice. At the end of each treatment period, samples of unstimulated saliva and dental biofilm were collected $8 \mathrm{~h}$ after the last oral hygiene procedure. Fluoride concentrations in saliva and dental biofilm were analyzed using a specific electrode. The fluoride concentrations in saliva and dental biofilm $8 \mathrm{~h}$ after the last use of fluoride products did not differ among treatments. The results of this study suggest that treatments with home-use fluoride products have no long-term effect on fluoride concentrations in saliva and in dental biofilm of residents of an area with a fluoridated water supply.
\end{abstract}

Keywords: Fluorine; Toothpastes; Mouthwashes; Saliva; Biofilms.

\section{Introduction}

Fluoride is thought to exert anti-caries effects predominantly by elevating the fluoride concentrations in saliva and dental biofilm. ${ }^{1}$ Fluoride inhibits acid-mediated dissolution of tooth mineral and promotes remineralization of the crystal surface. ${ }^{1}$

The greatest advantage of fluoride dentifrices, compared to other forms of topical application, is the regularity with which fluoride is delivered, which increases the availability of fluoride in the oral cavity during the caries process. ${ }^{2}$ Regular use of fluoride dentifrices is thought to be a beneficial preventive measure, regardless of the caries experience or other oral healthcare measures. ${ }^{3}$ However, for patients with active caries, a greater frequency of topical fluoride administration is needed. ${ }^{4}$ Mouthrinsing is a simple method of self-application of fluoride, ${ }^{5}$ usually recommended for patients with active caries in addition to the normal use of a fluoride dentifrice. ${ }^{5}$ However, because a mouthrinse is usually applied immediately after the toothbrush procedure, it does not increase the frequency of fluoride exposure. ${ }^{6}$

Some studies have suggested that the daily, regular use of home-use topical fluoride products, e.g., dentifrices and mouthrinses, results in 
dental exposure to low and steady fluoride concentrations via oral fluids. ${ }^{7,8}$ An increase in the fluoride concentration in saliva from 0.01 to $0.1 \mathrm{ppm}$, i.e., a 5- to 10-fold increase, for prolonged periods may be very effective for caries control. ${ }^{9}$

The concentration of fluoride in dental biofilm increases with the use of fluoride dentifrices, ${ }^{10}$ fluoride mouthrinses, ${ }^{11}$ and fluoridated drinking water. ${ }^{12}$ The fluoride concentration present in oral fluids at any given time following fluoride application is influenced by the initial concentration of the fluoride applied, by the elapsed time since the last exposure, by the method of delivery, and by a complex interaction among factors that influence fluoride clearance and retention. ${ }^{13}$ Duckworth and Morgan ${ }^{14}$ stated that after a single brushing with a fluoride dentifrice, the salivary fluoride content decreases in two distinct phases: an initial, rapid phase that lasts for 40-80 $\mathrm{min}$ depending on the individual; and a second, slower phase that lasts for several hours because fluoride is released from an oral fluoride reservoir.

A majority of studies that have evaluated fluoride concentrations in saliva after treatment with topical fluoride have observed that the concentrations decreased shortly after a single application. ${ }^{15,16,17,18,19}$ Numerous studies have determined fluoride concentrations in saliva and in dental biofilm over long periods in areas with fluoridated water supply; however, they yielded contradictory results. ${ }^{13,20,21,22,23,24}$

Since the literature remains inconclusive in regard to this aspect, the aim of this study was to determine the fluoride retention in saliva and in dental biofilm after different home-use fluoride treatments in volunteers living in an area with a fluoridated water supply.

\section{Methodology}

Dental students $(23.4 \pm 3.6$ years old $)$ residing in an area with a fluoridated water supply (mean: 0.7 ppm F, range: $0.6-0.8$ ppm F) were recruited at the Faculty of Dentistry of Universidade Federal do Rio Grande do Sul (UFRGS), Brazil. The sample size calculation was performed using dental biofilm fluoride concentration data from a previous study by Heijnsbroek et al. ${ }^{25}$ Using a similar experimental protocol, incorporating $20 \%$ beta and $5 \%$ alpha errors with a statistic power of 0.8 , the required sample size was calculated to be 31 volunteers. Assuming a dropout rate of 30\%, 40 volunteers were enrolled. The inclusion criteria were the following: good general and dental health, no use of antibiotics at least 2 months prior to the study, at least 24 natural teeth, and an unstimulated salivary flow rate of $0.25-0.35$ $\mathrm{mL} / \mathrm{min} .{ }^{20}$ The study was approved by the Ethics Committee of the Faculty of Dentistry, UFRGS (protocol number 53/06), and the volunteers provided informed consent.

This study used a single-blind, randomized, crossover design. Volunteers were assigned randomization numbers using a computer-generated allocation schedule. Professional dental prophylaxis was performed before and during the experimental periods in order to avoid a carryover effect from previous treatments. The volunteers were instructed to use a fluoride-free dentifrice (silicabased, Dentics ${ }^{\circledR}$, DentalPrev Ind. e Com. Ltda., São Paulo, Brazil) for 1 week prior to the study and during the washout periods. The volunteers were administered four different treatments, each lasting 1 week: T I: twice-daily placebo dentifrice, T II: twice-daily fluoride dentifrice, T III: twicedaily fluoride dentifrice and once-daily fluoride mouthrinse, and T IV: thrice-daily fluoride dentifrice. Each treatment was followed by a 1-week washout period. The volunteers brushed their teeth with the dentifrice (placebo or 1100 ppm F as NaF, silica-based, Dentics ${ }^{\circledR}$ ) for $1 \mathrm{~min}$ and then rinsed the mouth with $10 \mathrm{~mL}$ of tap water for $10 \mathrm{~s}$. The rinsing procedure with the fluoride solution (225 ppm $\mathrm{F}$ as $\mathrm{NaF}$, Dentics ${ }^{\circledR}$ ) was performed with $10 \mathrm{~mL}$ solution for $1 \mathrm{~min}$. The volunteers brushed their teeth in the morning and at bedtime. In treatment IV, the volunteers brushed their teeth at lunchtime as well. The rinsing procedure was performed immediately after bedtime toothbrushing. The volunteers received oral and written information about the procedures and were asked to refrain from using any antibacterial or other fluoridated agents during the experimental period. Owing to the crossover design, the volunteers did not receive any instructions regarding their daily diet. In an attempt to optimize compliance, all volunteers 
received a phone call before the sampling procedures to remind them of their scheduled time for their last oral hygiene procedure.

Eight hours after the last oral hygiene procedure, in the morning (before breakfast), dental biofilm samples were collected with a sterile curette from the supragingival margins of molar and pre-molar buccal surfaces. The samples were placed in Eppendorf tubes that were codified and pre-weighted on an analytical balance (Sartorius, Goettingen, Germany). A pilot study previously determined the minimum amount of dental biofilm required for fluoride analysis. At least $2 \mathrm{mg}$ of dental biofilm was collected in each phase from each volunteer. The collected dental biofilm samples were immediately centrifuged for $2 \mathrm{~min}$ at 11,000 $\times \mathrm{g}$ (Eppendorf, Hamburg, Germany). ${ }^{25}$ The volunteers were instructed to drool for $5 \mathrm{~min}$ in a plastic cup to obtain unstimulated whole saliva samples. The saliva samples were placed in Eppendorf tubes, centrifuged for $15 \mathrm{~min}$ at $11,000 \times g$, and the supernatants were frozen for subsequent fluoride analysis. ${ }^{25}$

In order to determine the fluoride content, calibration curves for saliva and dental biofilm were drawn from standardized solutions containing known amounts of fluoride, ranging from 0.02-0.4 ppm F and 0.1-16 ppm F, respectively. All the samples were analyzed using an ion-specific electrode (Orion Research Inc., Beverly, USA) connected to an ion analyzer (Procyon, São Paulo, Brazil). Sample readings were recorded in millivolts $(\mathrm{mV})$ and transformed by linear regression of the calibration curve into fluoride concentrations of $\mu \mathrm{mol} / \mathrm{L}$ and $\mathrm{mmol} / \mathrm{kg}$ (saliva and dental biofilm, respectively). ${ }^{18,26}$

Before analyses, the dental biofilm samples were dehydrated for $24 \mathrm{~h}$ in a vacuum dryer over $\mathrm{P}_{2} \mathrm{O}_{5}{ }^{27}$ For fluoride extraction, $0.5 \mathrm{M} \mathrm{HCl}$ was added to the microtube at $0.1 \mathrm{~mL} \mathrm{HCl}$ per mg biofilm (dry weight). After extraction for $3 \mathrm{~h}$ at room temperature under constant agitation, an equal volume of Total Ionic Strength Adjustment Buffer (TISAB) II, pH 5.0, modified with $20 \mathrm{~g} \mathrm{NaOH} / \mathrm{L}$, was added to the microtube. The samples were centrifuged for $10 \mathrm{~min}$ at $11,000 \times$ $g$ and the supernatant was collected for determination of the fluoride concentration. . $7,28,29^{2}$

Saliva samples were thawed and mixed with TISAB III (Thermo Electron Corp., Beverly, USA) at a 10:1 ratio for determination of the fluoride content. ${ }^{11}$

The non-parametric Friedman's test was used to compare the fluoride concentrations in saliva and in dental biofilm after the different treatments. Because the trial had a crossover design, each volunteer was considered as an experimental unit. All of statistical analyses were performed using SAS version 9.1 (SAS Institute Inc., Cary, USA). The level of significance was set at 5\%.

\section{Results}

Two volunteers were excluded because they failed to attend one or more appointments and their saliva and dental biofilm samples were not included in the data analysis. Table 1 shows the fluoride concentrations of saliva and dental biofilm samples at $8 \mathrm{~h}$ after the end of each treatment. No differences in the fluoride concentrations in saliva or in dental biofilm were observed among the different treatments $(\mathrm{p}>0.05)$.

Table 1. Fluoride concentrations in saliva and in dental biofilm after different treatments

\begin{tabular}{|c|c|c|c|c|c|c|c|c|c|}
\hline \multirow[b]{3}{*}{ Treatments* } & \multicolumn{5}{|c|}{ Saliva ( $\mu \mathrm{mol} F / L)$} & \multicolumn{4}{|c|}{ Dental biofilm (mmol F/kg) } \\
\hline & & \multicolumn{4}{|c|}{ Percentiles } & & \multicolumn{3}{|c|}{ Percentiles } \\
\hline & $\mathrm{n}$ & Mean (SD) & $25^{\text {th }}$ & $50^{\text {th }}$ & $75^{\text {th }}$ & Mean (SD) & $25^{\text {th }}$ & $50^{\text {th }}$ & $75^{\text {th }}$ \\
\hline $\mathrm{TI}$ & 38 & $1.02(0.53)$ & 0.75 & 0.84 & 1.08 & $13.88(15.65)$ & 2.81 & 5.30 & 22.46 \\
\hline $\mathrm{T} \|$ & 38 & $0.99(0.27)$ & 0.79 & 0.95 & 1.21 & $16.81(27.70)$ & 3.92 & 7.26 & 19.07 \\
\hline T III & 38 & $1.08(0.43)$ & 0.79 & 1.00 & 1.34 & $18.53(21.42)$ & 3.63 & 10.65 & 25.06 \\
\hline \multirow[t]{2}{*}{ TIV } & 38 & $1.11(0.53)$ & 0.84 & 0.95 & 1.24 & $18.80(18.88)$ & 4.31 & 10.98 & 30.70 \\
\hline & \multicolumn{6}{|c|}{$p=0.49^{* *}$} & \multicolumn{3}{|c|}{$p=0.26^{* *}$} \\
\hline
\end{tabular}

*T I: twice-daily placebo dentifrice; T II: twice-daily fluoride dentifrice; $T$ III: twice-daily fluoride dentifrice and once-daily fluoride mouthrinse; $T$ IV: thrice-daily fluoride dentifrice

** Friedman test 


\section{Discussion}

In this study, we evaluated whether fluoride concentrations in saliva and dental biofilm remained significantly elevated at $8 \mathrm{~h}$ after four different oral hygiene procedures in volunteers living in an area with fluoridated water supply. The fluoride concentrations in saliva and in dental biofilm were not significantly different among the treatments. The frequency of brushing with a fluoride dentifrice and additional use of fluoride mouthrinse followed by brushing with a fluoride dentifrice did not affect the fluoride concentrations in biofilm and saliva at $8 \mathrm{~h}$ after the last procedure.

Our results did not confirm those of others studies, which evaluated fluoride retention after use of fluoride products. $7,8,14,15,25,26,30$ In these studies, fluoride measurements were taken on saliva samples collected 6-24 h after the regular daily use of fluoride products, brushing with a fluoride dentifrice, and/or using fluoride mouthrinsing. Most of these studies reported that the fluoride concentrations in saliva remained significantly elevated, over a period of 12-24 h, compared with the baseline. One possible explanation for the results of the present study could be that previous studies were performed in areas without fluoridated drinking water. This could have resulted in greater fluoride concentrations in saliva and in dental biofilm at $12 \mathrm{~h}$ or more after the last use of fluoride products, as compared with a relatively low baseline concentration. ${ }^{30}$

The results of this study are in agreement with the findings of most of the studies conducted in areas with a fluoridated water supply. ${ }^{13,20,21,22}$ These studies also did not show a long-term effect on the fluoride concentration in saliva and in dental biofilm compared to baseline values. A possible explanation for these is that plaque-binding sites for long-term fluoride retention are occupied by fluoride ions largely in communities with fluoridated water, but not where the water contains only traces of this ion. ${ }^{30}$ However, two studies carried out by the same authors and conducted in an area with a fluoridated water supply yielded contradictory results. ${ }^{22,24}$ The results of the study conducted in $2006^{22}$ are in agreement with our findings; no differences in the fluoride concentrations in saliva and in dental biofilm were noted at 12 hours after the use of fluoridated toothpaste when compared to baseline values. However, in the study carried out in 2010, ${ }^{24}$ the authors found that the fluoride concentrations in saliva and in dental biofilm remained elevated at 12 $\mathrm{h}$ after the last use of a fluoride dentifrice. A possible explanation for these contradictory results could be the different fluoride concentrations in the water supply of the city where the studies were conducted. In the study from 2006, the mean fluoride concentration in the water supply was $0.85 \mathrm{ppm}$, whereas in the study from 2010, the mean fluoride concentration was 0.72 $\mathrm{ppm}$. This small difference between the fluoride concentrations in water observed in the two studies may have had an impact on having or not having the oral binding sites for fluoride occupied by fluoride ions.

Limitations of this study could be the time of measurement of the fluoride concentrations in biofilm and saliva for the different treatments, since this study was conducted in an area with fluoridated water supply. Nevertheless, analyzing the medians and $25^{\text {th }}-75^{\text {th }}$ percentile values for dental biofilm obtained from the the different treatments, the treatment with twice-daily placebo dentifrice (T I) showed the lowest median and $25^{\text {th }}-75^{\text {th }}$ percentile values followed by the treatment with twice-daily fluoride dentifrice (T II). The treatments with greater frequency and higher dose of fluoride products (T III: twice-daily fluoride dentifrice and once-daily fluoride mouthrinse and T IV: thricedaily fluoride dentifrice) showed higher median and $25^{\text {th }}-75^{\text {th }}$ percentile values. A shorter measurement time interval (less than $8 \mathrm{~h}$ ) may reveal significant differences among the treatments.

\section{Conclusion}

The results of this study suggest that treatments with home-use fluoride products have no long-term effect on fluoride concentrations in saliva and in dental biofilm of residents of an area with a fluoridated water supply.

\section{Acknowledgments}

The authors thank the volunteers at the Faculty of Dentistry of Universidade Federal do Rio Grande do Sul (UFRGS) for their valuable participation in this study; DentalPrev Ind. e Com. Ltda., Brazil, for dentifrice and mouthrinse preparation and donation; and Coordenação de Aperfeiçoamento de Pessoal de Nível Superior (CAPES) and Pró-Reitoria de Pesquisa of UFRGS for the financial support. 


\section{References}

1. ten Cate JM. Current concepts on the theories of the mechanism of action of fluoride. Acta Odontol Scand. 1999 Dec;57(6):325-9.

2. Mellberg JR, Chomicki WG. Effect of soluble calcium on fluoride uptake by artificial caries lesions in vivo. Caries Res. 1985;19(2):122-5.

3. Delbem ACB, Carvalho LPR, Morihisa RKU, Cury JA. In vitro comparison of the cariostatic effect between topical application of fluoride gels and fluoride toothpaste. J Appl Oral Sci. 2004 Jun;12(2):121-6.

4. Marinho VC, Higgins JP, Sheiham A, Logan S. Fluoride toothpastes for preventing dental caries in children and adolescents. Cochrane Database Syst Rev. 2003;(1):CD002278.

5. Marinho VC, Higgins JP, Logan S, Sheiham A. Fluoride mouthrinses for preventing dental caries in children and adolescents. Cochrane Database Syst Rev. 2003;(3):CD002284.

6. Marinho VC, Higgins JP, Sheiham A, Logan S. Combinations of topical fluoride (toothpastes, mouthrinses, gels, varnishes) versus single topical fluoride for preventing dental caries in children and adolescents. Cochrane Database Syst Rev. 2004;(1):CD002781.

7. Duckworth RM, Gilbert RJ. Intra-oral models to assess cariogenicity: evaluation of oral fluoride and $\mathrm{pH}$. J Dent Res. 1992 Apr;71 Spec No:934-44.

8. Duckworth RM, Morgan SN, Gilbert RJ. Oral fluoride measurements for estimation of the anti-caries efficacy of fluoride treatments. J Dent Res. 1992 Apr;71 Spec No:836-40.

9. Featherstone JD, Zero DT. An in situ model for simultaneous assessment of inhibition of demineralization and enhancement of remineralization. J Dent Res. 1992 Apr;71 Spec No:804-10.

10. Duckworth RM, Morgan SN, Burchell CK. Fluoride in plaque following use of dentifrices containing sodium monofluorophosphate. J Dent Res. 1989 Feb;68(2):130-3.

11. 11.Duckworth RM, Morgan SN, Murray AM. Fluoride in saliva and plaque following use of fluoride-containing mouthwashes. J Dent Res. 1987 Dec;66(12):1730-4.

12. Santos MN, Cury JA. Dental plaque fluoride is lower after discontinuation of water fluoridation. Caries Res. 1988;22(5):316-7.

13. Zero DT, Fu J, Espeland MA, Featherstone JD. Comparison of fluoride concentrations in unstimulated whole saliva following the use of a fluoride dentifrice and a fluoride rinse. J Dent Res. 1988 Oct;67(10):1257-62.

14. Duckworth RM, Morgan SN. Oral fluoride retention after use of fluoride dentifrices. Caries Res. 1991;25(2):123-9.

15. Duckworth RM, Knoop DT, Stephen KW. Effect of mouthrinsing after toothbrushing with a fluoride dentifrice on human salivary fluoride levels. Caries Res. 1991;25(4):287-91.

16. Seppa L, Salmenkivi S, Hausen H. Salivary fluoride concentration in adults after different fluoride procedures. Acta Odontol Scand. 1997 Apr;55(2):84-7.
17. Sjogren K, Birkhed D. Effect of various post-brushing activities on salivary fluoride concentration after toothbrushing with a sodium fluoride dentifrice. Caries Res. 1994;28(2):127-31.

18. Issa AI, Toumba KJ. Oral fluoride retention in saliva following toothbrushing with child and adult dentifrices with and without water rinsing. Caries Res. 2004 Jan-Feb;38(1):15-9.

19. Sjogren K, Melin NH. The influence of rinsing routines on fluoride retention after toothbrushing. Gerodontology. 2001 Jul;18(1):15-20.

20. Zero DT, Raubertas RF, Fu J, Pedersen AM, Hayes AL, Featherstone JD. Fluoride concentrations in plaque, whole saliva, and ductal saliva after application of home-use topical fluorides [published eerratum appears in J Dent Res 1993 Jan;72(1):87]. J Dent Res. 1992 Nov;71(11):1768-75.

21. Whitford GM, Wasdin JL, Schafer TE, Adair SM. Plaque fluoride concentrations are dependent on plaque calcium concentrations. Caries Res. 2002 Jul-Aug;36(4):256-65.

22. Pessan JP, Sicca CM, de Souza TS, da Silva SM, Whitford GM, Buzalaf MA. Fluoride concentrations in dental plaque and saliva after the use of a fluoride dentifrice preceded by a calcium lactate rinse. Eur J Oral Sci. 2006 Dec;114(6):489-93.

23. Pessan JP, Silva SM, Lauris JR, Sampaio FC, Whitford GM, Buzalaf MA. Fluoride uptake by plaque from water and from dentifrice. J Dent Res. 2008 May;87(5):461-5.

24. Pessan JP, Alves KM, Ramires I, Taga MF, Sampaio FC, Whitford GM, Buzalaf MA. Effects of regular and lowfluoride dentifrices on plaque fluoride. J Dent Res. 2010 Oct;89(10):1106-10.

25. Heijnsbroek M, Gerardu VA, Buijs MJ, van Loveren C, ten Cate JM, Timmerman MF, et al. Increased salivary fluoride concentrations after post-brush fluoride rinsing not reflected in dental plaque. Caries Res. 2006;40(5):444-8.

26. Campus G, Lallai MR, Carboni R. Fluoride concentration in saliva after use of oral hygiene products. Caries Res. 2003 Jan-Feb;37(1):66-70.

27. Pearce EI, Hancock EM, Gallagher IH. The effect of fluorhydroxyapatite in experimental human dental plaque on its $\mathrm{pH}$, acid production and soluble calcium, phosphate and fluoride levels following glucose challenge. Arch Oral Biol. 1984;29(7):521-7.

28. Cury JA, Rebelo MA, Del Bel Cury AA, Derbyshire MT, Tabchoury CP. Biochemical composition and cariogenicity of dental plaque formed in the presence of sucrose or glucose and fructose. Caries Res. 2000 Nov-Dec;34(6):491-7.

29. Tatevossian A. Fluoride in dental plaque and its effects. J Dent Res. 1990 Feb;69 Spec No:645-52; discussion 82-3.

30. Whitford GM, Buzalaf MA, Bijella MF, Waller JL. Plaque fluoride concentrations in a community without water fluoridation: effects of calcium and use of a fluoride or placebo dentifrice. Caries Res. 2005 Mar-Apr;39(2):100-7. 